\title{
Flexible modeling improves assessment of prognostic value of C-reactive protein in advanced non-small cell lung cancer
}

\author{
B Gagnon $^{*, 1}$, M Abrahamowicz ${ }^{2}$, Y Xiao ${ }^{2}$, M-E Beauchamp ${ }^{2}$, N MacDonald $^{3}$, G Kasymjanova $^{4}$, H Kreisman $^{4}$ and $^{4}$ \\ D Small ${ }^{5}$
}

'Department of Medicine and Oncology, McGill University, 687 Pine Avenue West, R4.29, Montreal, Quebec, H3A IAI, Canada; ${ }^{2}$ Department of Epidemiology and Biostatistics, McGill University, 687 Pine Avenue West, V2, Montreal, Quebec, H3A IAI, Canada; ${ }^{3}$ Department of Oncology, Cancer Nutrition and Rehabilitation Program, McGill University, 687 Pine Avenue West, Montreal, Quebec, H2A IAI, Canada; ${ }^{4}$ Department of Internal Medicine, Division of Pulmonary Diseases, Sir Mortimer B Davis, Jewish General Hospital, 3755 Cote-Ste-Catherine, Montreal, Quebec, H3T IE2, Canada; ${ }^{5}$ Departments of Oncology and Internal Medicine, Division of Pulmonary Diseases, Sir Mortimer B Davis, Jewish General Hospital, 3755 Cote-Ste-Catherine, Montreal, Quebec, H3T IE2, Canada

BACKGROUND: C-reactive protein (CRP) is gaining credibility as a prognostic factor in different cancers. Cox's proportional hazard (PH) model is usually used to assess prognostic factors. However, this model imposes a priori assumptions, which are rarely tested, that ( $\mathrm{I}$ ) the hazard ratio associated with each prognostic factor remains constant across the follow-up (PH assumption) and (2) the relationship between a continuous predictor and the logarithm of the mortality hazard is linear (linearity assumption).

METHODS: We tested these two assumptions of the Cox's PH model for CRP, using a flexible statistical model, while adjusting for other known prognostic factors, in a cohort of 269 patients newly diagnosed with non-small cell lung cancer (NSCLC).

RESULTS: In the Cox's PH model, high CRP increased the risk of death (HR= I. I I per each doubling of CRP value, 95\% Cl: I.03- I.20, $P=0.008)$. However, both the $\mathrm{PH}$ assumption $(P=0.033)$ and the linearity assumption $(P=0.0 \mathrm{I} 5)$ were rejected for $C R P$, measured at the initiation of chemotherapy, which kept its prognostic value for approximately 18 months.

CONCLUSION: Our analysis shows that flexible modeling provides new insights regarding the value of CRP as a prognostic factor in NSCLC and that Cox's PH model underestimates early risks associated with high CRP.

British Journal of Cancer (20 I0) I 02, I I I3- I I22. doi: I0.1038/sj.bjc.6605603 www.bjcancer.com

Published online 16 March 2010

(c) 2010 Cancer Research UK

Keywords: non-small cell lung cancer; inflammation; prognosis; C-reactive protein; albumin; survival analysis

Patients with advanced non-small cell lung cancer (NSCLC) have a grim prognosis; most will die in less than a year (Schrump et al, 2008). Identifying patients at higher risk of very short survival times is important for optimal clinical management. Furthermore, the patients' understanding of their prognosis influences their willingness to receive life-extending therapy (Weeks et al, 1998a). Yet, estimation of expected survival times remains a clinically difficult (Glare, 2005; Watine et al, 2006; Kellett, 2008) and an emotionally challenging task (Berry, 2008).

In recent years, different biological markers have been suggested to improve prognostication of cancer (Mahmoud and Rivera, 2002; Maltoni et al, 2005). Among them, inflammatory markers, especially C-reactive protein (CRP), have been suggested to predict survival in different cancers (McMillan, 2008; Koch et al, 2009), including NSCLC (Kato et al, 2000; Forrest et al, 2003, 2004). In these published studies of NSCLC mortality (Kato et al, 2000; Forrest et al, 2003, 2004), the prognostic value of CRP was assessed using the Cox's proportional hazard (PH) model (Cox, 1972).

To improve both prognosis and clinical management, an accurate assessment of the independent relationships between

*Correspondence: Dr B Gagnon; E-mail: bruno.gagnon@clinepi.mcgill.ca Received 17 August 2009; revised 8 February 2010; accepted 15 February 2010; published online 16 March 2010 putative prognostic factors, such as CRP, and mortality is paramount. To ensure valid conclusions and accurate risk prediction, prognostic studies should rely on statistical methods that correctly represent the actual structure of empirical data and the true complexity of the biological processes under study. From this perspective, it is imperative to verify the assumptions underlying the statistical models to be used in the analysis (Altman et al, 1995). The Cox's PH model imposes the assumption that the mortality hazards associated with different patterns of covariates ('risk profiles') are proportional, which implies that the estimated effects of prognostic factors on the hazard are a priori constrained to remain constant over the entire follow-up time (Cox, 1972). This crucial assumption is rarely tested in prognostic studies of cancer mortality (Altman et al, 1995). Yet, in different cancers, it has been shown to be inconsistent with the actual effect of various prognostic factors, whose effect on mortality did change over time (Gray, 1992; Hess, 1994; Kooperberg et al, 1995; Rachet et al, 1998; Quantin et al, 1999; Lambert et al, 2005; Remontet et al, 2007; Binquet et al, 2009). In such situations, an inappropriate use of the Cox's PH model may lead to biased results, inaccurate prediction, loss of statistical power, and incorrect conclusions (Altman et al, 1995; Abrahamowicz et al, 1996).

Furthermore, conventional statistical models, such as the Cox's $\mathrm{PH}$ model, rely on the linearity assumption, according to which the relationships between continuous prognostic factors and the 
respective outcome measure (logarithm of the hazard in the case of Cox's PH model) are linear. This would imply that, for example, the relative risk of mortality is the same when comparing (a) an 80year old $v s$ a 60 -year-old subject, and (b) a 40-year old $v s$ a 20 -year old, because in both cases there is a 20 -year age difference. Again, in the last two decades, several epidemiological and clinical studies have shown that the linearity assumption is seriously violated for many prognostic and risk factors, and its a priori acceptance may lead to important biases and misleading conclusions (Hastie and Tibshirani, 1990; Sleeper and Harrington, 1990; Gray, 1992; Royston and Altman, 1994; Abrahamowicz et al, 1997; Remontet et al, 2007; Royston and Sauerbrei, 2008). Thus, the methodological arguments and the empirical evidence indicate that both the $\mathrm{PH}$ and the linearity assumptions should be carefully verified in prognostic studies.

Our aim is to re-assess the ability of CRP to predict survival in a cohort of patients recently diagnosed with non-resectable NSCLC before receiving chemotherapy. To this end, we used the flexible generalisation of the Cox's model, which permits testing the conventional $\mathrm{PH}$ and linearity hypotheses, and avoids imposing the a priori assumptions underlying the Cox's PH model. If these hypotheses were rejected, non-proportional and/or nonlinear effects of particular prognostic factors were estimated (Abrahamowicz and MacKenzie, 2007).

\section{PATIENTS AND METHODS}

\section{Patients}

Since May 2001, clinical data of patients with lung cancer, observed at the Jewish General Hospital Pulmonary Oncology Clinic (POC) in Montreal, Quebec, Canada, were prospectively recorded in a computerised database. These data included, among other characteristics, age, sex, stage, ECOG performance status (Oken et al, 1982), smoking status, type of first-line chemotherapy regimen, and the blood parameters listed in Table 1 . The level of CRP started to be measured in January 2002 by one POC physician and, after January 2005, by all POC physicians. A majority of the blood tests were taken within 3 days before the first chemotherapy dose.

For the purpose of this study, we extracted the data for patients treated between 9 April 2002 and 18 September 2008, and terminated their follow-up on 15 March 2009. Dates of death were obtained from clinical charts. The study was approved by the Institutional Review Board of the Jewish General Hospital.

\section{Statistical analyses}

Data analyses Descriptive statistics were used to compare the baseline characteristics of subjects for whom CRP was available and who were, thereby, included in the analyses, $v s$ those excluded.

In the main analyses, we used multivariable survival analytical methods for right-censored time-to-event data (Cox, 1972). Time 0 was defined as the date of the first chemotherapy treatment and the event of interest was death because of any cause. Patients who were alive at the end of the study, on 15 March 2009, were censored. In preliminary analyses, we assessed the distributions of continuous variables and used a logarithmic transformation with base 2 for the only two variables with considerable positive skewness: CRP and lactate dehydrogenase (LDH).

Statistical models Two different types of survival analytical models were used. First, we used both the univariate and the multivariable Cox's PH models (Cox, 1972), which a priori imposed the $\mathrm{PH}$ assumption and, for continuous covariates, the linearity assumption.

The second model represented a flexible generalisation of the Cox's model, recently developed and validated by Abrahamowicz
Table I Patients' characteristics $(N=269)^{\mathrm{a}}$

\begin{tabular}{|c|c|}
\hline Variables & Descriptive statistics \\
\hline Age: mean (s.d.) & $64.3(11.0)$ \\
\hline Sex: female $n(\%)$ & $136(50.6)$ \\
\hline Stage: IIIA/IIIB n (\%) & $70(26.0)$ \\
\hline IIIB+pleural effusion/IV n (\%) & $199(74.0)$ \\
\hline \multicolumn{2}{|l|}{ ECOG $^{\text {b }}$ performance status: } \\
\hline $0-\ln (\%)$ & $213(79.2)$ \\
\hline $2 n(\%)$ & $56(20.8)$ \\
\hline Smoking status: Never n (\%) & $4 \mid(15.2)$ \\
\hline Ever n (\%) & $228(84.8)$ \\
\hline \multicolumn{2}{|l|}{ Chemotherapy type: } \\
\hline Single-agent $n(\%)$ & $66(24.5)$ \\
\hline Double-agent $n(\%)^{c}$ & $203(75.5)$ \\
\hline \multicolumn{2}{|l|}{ Pathology: } \\
\hline Adenocarcinoma $n(\%)$ & $177(65.8)$ \\
\hline Large cell carcinoma $n(\%)$ & $22(8.2)$ \\
\hline Squamous cell carcinoma $n$ (\%) & $32(11.9)$ \\
\hline Undifferentiated carcinoma $n$ (\%) & $38(14.1)$ \\
\hline \multicolumn{2}{|l|}{ CRP': } \\
\hline Mean (s.d.) & $36.2(53.6)$ \\
\hline Median \{quartile\} (range) & I3.I $\{4.9,39.9\}(0.3,3 \mid 6.8)$ \\
\hline \multicolumn{2}{|l|}{$\log _{2}$ CRP: } \\
\hline Mean (s.d.) & $3.8(2.2)$ \\
\hline Median \{quartile\} (range) & $3.7\{2.3,5.3\}(-1.7,8.3)$ \\
\hline \multicolumn{2}{|l|}{ Albumin ${ }^{d}:$} \\
\hline Mean (s.d.) & $40.2(4.1)$ \\
\hline \multicolumn{2}{|l|}{$\mathrm{LDH}^{\mathrm{d}}:$} \\
\hline Mean (s.d.) & $248.8(199.4)$ \\
\hline Median \{quartile\} (range) & $211\{169,263\}(98,2500)$ \\
\hline \multicolumn{2}{|l|}{$\log _{2}$ LDH: } \\
\hline Mean (s.d.) & $7.8(0.6)$ \\
\hline Median \{quartile\} (range) & $7.7\{7.4,8.0\}(6.6,11.3)$ \\
\hline Calciumm: mean (s.d.) & $2.35(0.19)$ \\
\hline Alkaline phosphatase ${ }^{\text {d: }}$ mean (s.d.) & $104.2(59.2)$ \\
\hline Haemoglobin : mean (s.d.) & $130.2(17.1)$ \\
\hline Platelet: mean (s.d.) & $326.7($ ( I 17.2) \\
\hline Neutrophil counts: mean (s.d.) & $7.09(3.55)$ \\
\hline Lymphocytes ${ }^{d}$ : mean (s.d.) & $1.59(0.70)$ \\
\hline Percentage of weight loss: mean (s.d.) & $4.9(6.6)$ \\
\hline
\end{tabular}

Abbreviations: $\mathrm{CRP}=\mathrm{C}$-reactive protein; $\mathrm{LDH}=$ lactate dehydrogenase. ${ }^{\mathrm{a}} \mathrm{N}=269$

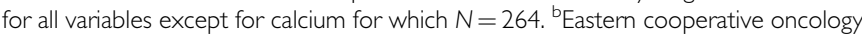
group. 'All include platinum-based chemotherapy. ${ }^{\mathrm{d}}$ Normal values: CRP $\leqslant 10 \mathrm{mgl}^{-1}$; albumin 35-52 $\mathrm{gl}^{-1}$; LDH $110-210 \mathrm{UI}^{-1}$; calcium $2.12-2.62 \mathrm{mmoll}^{-1}$; alkaline phosphatase 56-120 $\mathrm{UI}^{-1}$; haemoglobin $140-\left.180 \mathrm{~g}\right|^{-1}$; platelet $140-440 \times 10^{9} \mathrm{I}^{-1}$; neutrophil counts $1.60-7.70 \times 10^{9} \mathrm{I}^{-1}$; and lymphocytes $0.80-4.40 \times 10^{9} \mathrm{I}^{-1}$.

and MacKenzie (Abrahamowicz and MacKenzie, 2007). The general idea of flexible modeling is to avoid restrictive a priori assumptions underlying standard statistical models and model the effects of prognostic factors through flexible functions, the shapes of which are estimated directly from the data, rather than being imposed by the model. Such flexible models use various, typically computationally intensive, statistical techniques, such as regression splines (Ramsay, 1988), generalised additive models (Hastie and Tibshirani, 1990), or fractional polynomials (Royston and Sauerbrei, 2008). The flexible model used in our analyses uses quadratic regression splines, that is, piecewise quadratic polynomials that can recover a large variety of clinically plausible functions and, thus, accurately estimate both non- $\mathrm{PH}$ and nonlinear effects of continuous predictors (Abrahamowicz and 
MacKenzie, 2007). Throughout the paper, we use the term 'flexible spline-based model' to refer to the flexible generalisation of the Cox's PH model that uses quadratic regression splines to model time-dependent and/or non-linear covariate effects with quadratic regression splines, as in (Abrahamowicz and MacKenzie, 2007).

The flexible spline-based model allowed us to test, for each continuous prognostic factor, the two assumptions underlying the Cox's PH model: (a) the PH hypothesis that the effect of the predictor remained constant over time and (b) the linearity of the effect on the log hazard (linearity hypothesis). If one or both of these assumptions were rejected for a given prognostic factor, then the flexible spline-based model permitted an accurate re-modeling of its effect on the hazard. Specifically, if the PH hypothesis was rejected, with $P<0.05$ for a non-parametric model-based likelihood ratio test (LRT), then splines were used to model the timedependent hazard ratio (HR), that is, to estimate how the strength of the predictor's effect on the hazard changes with increasing follow-up time (Abrahamowicz and MacKenzie, 2007). Conversely, if the linearity hypothesis was rejected, then splines were used to assess how the risk (log hazard) changed with increasing predictor value. If these hypotheses were not rejected $(P>0.05)$, then, to avoid over-fit bias (Abrahamowicz and Ciampi, 1991) and adhere to the model parsimony principle, the predictor effect was estimated as, respectively, constant-over-time HR and/or linear function. For binary predictors, only the $\mathrm{PH}$ hypothesis was tested and, if rejected, the time-dependent effect was estimated (Abrahamowicz et al, 1996).

Univariate analyses The analyses started with a series of separate, univariate Cox's PH models, each evaluating one of the following baseline characteristics, initially considered as potential prognostic factors for NSCLC mortality (Table 1): (a) categorical variables: sex, stage (IIIA and IIIB without pleural effusion $v s$ IIIB with pleural effusion and IV), performance status (ECOG 0-1 $v s$ 2), smoking history (ever $v s$ never), chemotherapy regimen (double vs single agents) and tumour pathology, and (b) continuous variables: age, log-transformed CRP ( $\log _{2} \mathrm{CRP}$ ), albumin, log-transformed LDH $\left(\log _{2} \mathrm{LDH}\right)$, calcium, alkaline phosphatise (ALP), haemoglobin, platelet, absolute neutrophil count (ANC), lymphocyte count, and percentage of weight lost.

Next, we estimated a series of univariate flexible spline-based models, each for a single prognostic factor, which tested the $\mathrm{PH}$ and, for continuous variables, the linearity assumptions, without adjustment for the other variables.

Strategies for building multivariable models Building a flexible multivariable model is challenging, especially if potential predictors are correlated (Sauerbrei et al, 2007). To build parsimonious final multivariable models, we used a multi-step procedure (Binquet et al, 2008). The flow chart in Figure 1 illustrates the consecutive steps of the analyses, explains how the results of the earlier steps affected the later steps, and identifies which results are presented in which table.

(1) At the first step, we first included all the aforementioned variables, listed in Table 1, in the multivariable Cox's $\mathrm{PH}$ model and then used the stepwise selection procedure, with $P<0.05$ and $P>0.05$ for the two-tailed Wald test as the criteria for, respectively, variables inclusion and elimination from the model. The selected variables were included in the final multivariable Cox's PH model. In addition, we have forced into the final Cox's PH model the performance status (ECOG 0-1 vs 2), even if it has a marginally nonsignificant effect in our analyses $(P=0.086)$, because it is an essential criterion for the treatment of unresectable NSCLC (Pfister et al, 2004).

(2) The next step of the multivariable model building process accounted for the fact that a variable may not be a significant predictor of survival in the Cox's $\mathrm{PH}$ model, which imposes a priori the $\mathrm{PH}$ and linearity assumptions, but may still have a significant time-dependent or non-linear effect (Abrahamowicz and MacKenzie, 2007). Therefore, the second step of the model building process involved fitting a series of separate flexible splinebased models, each of which tested the time-dependent and, for continuous variables, non-linear effects of a single variable, while adjusting for the PH-and-linear effects of all other variables selected at step 1 (Binquet et al, 2008). In other words, each model fitted at step 2 expanded the multivariable Cox's PH model built at step 1 by estimating and testing time-dependent and non-linear effects of a single predictor. On the basis of the results of the tests carried at the first and the second steps, we identified those variables that had statistically significant effects $(P<0.05)$ in at least one of the following: (a) Wald test of the association in the multivariable Cox's PH model selected by the stepwise selection in step 1, and/or (b) non-parametric LRT's of timedependent and/or non-linear effect(s) in the respective flexible

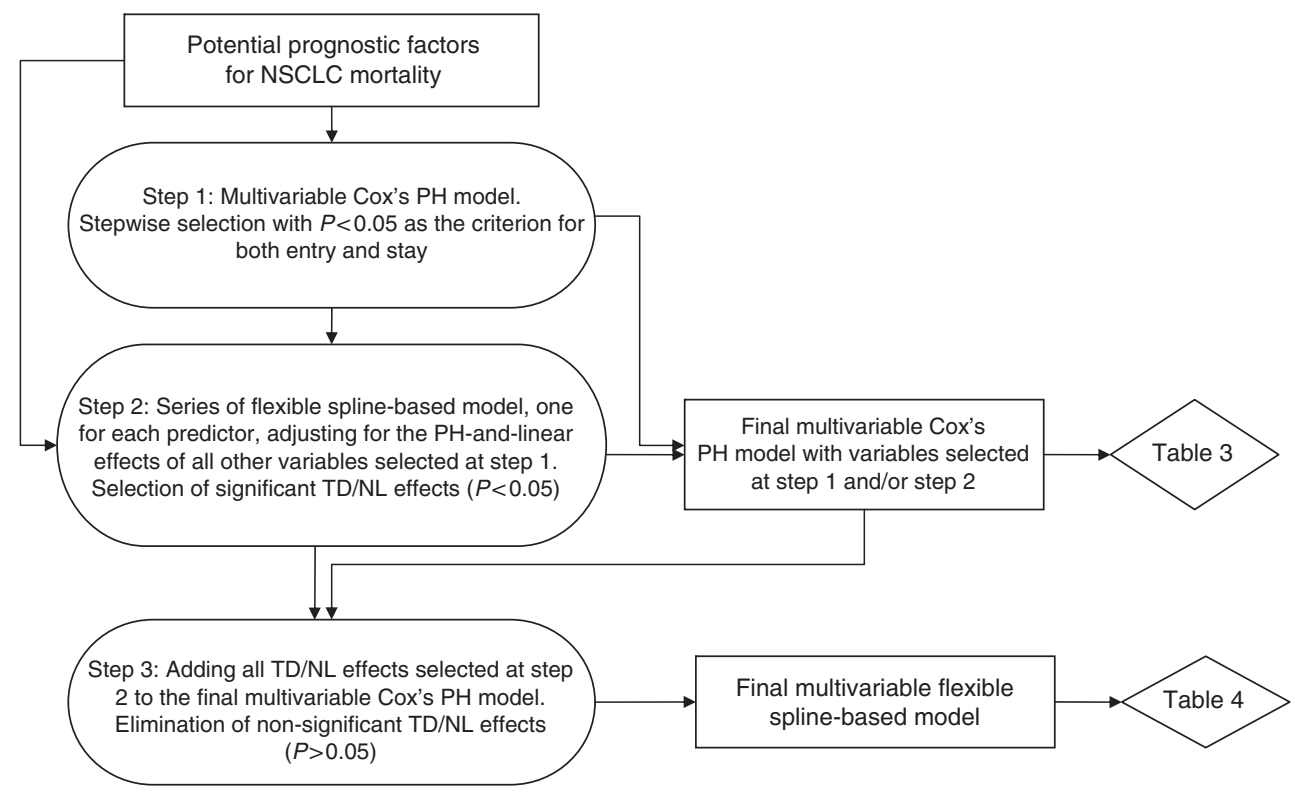

Figure I Flowchart of multivariable models building. 
spline-based model, estimated at step 2. Only those variables that met criteria (a) and/or (b) were included in the final multivariable versions of both Cox's PH model and the flexible spline-based model.

(3) To build the final multivariable flexible spline-based model, we first expanded the final Cox's PH model by including all 'nonparametric', that is, time-dependent and/or non-linear, effects that were statistically significant $(P<0.05)$ at step 2 . We then eliminated all those 'non-parametric' effects that became nonsignificant $(P>0.05)$ when adjusted for other 'non-parametric' effects as well as for all variables selected, and forced into the multivariable model, at steps 1 and 2 . As a result, the final multivariable flexible spline-based model included only those time-dependent and non-linear effects that remained statistically significant even when adjusted for each other, while all other variables selected for both final multivariable models were represented by parametric constant-over-time $(\mathrm{PH})$ linear effects (Binquet et al, 2008).

In the final flexible spline-based model, we tested the overall statistical significance of the effects of those continuous variables, which were represented by both non-linear and time-dependent functions. To this end, we used the 5-degree-offreedom $(\mathrm{df})$ non-parametric LRT that compared the deviance of the final flexible model with that of the corresponding reduced flexible model, from which a given variable was completely eliminated (Abrahamowicz and MacKenzie, 2007). The resulting $P$-value indicated the overall statistical significance of the independent association between the hazard and the variable, after having accounted for its non-linear and timedependent effects.

The goodness-of-fit of the Cox's PH model vs its flexible splinebased extension was compared with the Akaike's information criterion (AIC) (Akaike, 1974), which accounted for the increased complexity of the flexible spline-based model. A decrease of 10 or more AIC points indicates an important improvement in the model's predictive ability (Quantin et al, 1999).

Model validation To further compare the predictive ability of our final flexible spline-based model with that of the Cox's PH model, with the same predictors, we relied on the 'internal' crossvalidation procedure (Harrell, 2001). Specifically, we used the five-fold cross-validation algorithm (Rachet et al, 2003; Binquet et al, 2008), which involved splitting our sample of 269 patients into five randomly selected, mutually exclusive subsets of equal size. Then, the following two steps were repeated five times, separately for the Cox's PH and the flexible spline-based model. (1) One of the five subsets (validation subsample) was left out and the model was estimated using data from only the four other subsets. (2) The regression coefficients from step 1 were used to calculate the partial deviance for the respective validation subsample, that is, to assess how well the outcomes in that subsample were predicted by a given model (Binquet et al, 2008). Finally, the five deviance values, each from a different subset, were summed up to obtain the cross-validated partial deviance of the entire data set. As each subsetspecific deviance was calculated based on the model that did not depend on the data in the corresponding validation subset, the lower cross-validated deviance indicated the model expected to better predict the outcomes in an independent data set from a similar population (Rachet et al, 2003; Binquet et al, 2008).

Descriptive analyses and conventional Cox's $\mathrm{PH}$ regression were performed using the SAS statistical package (SAS Institute Inc., Cary, NC, USA), while the flexible spline-based model was implemented with a customised programme (Abrahamowicz and MacKenzie, 2007) written in the C programming language (Abrahamowicz et al, 1996).

\section{RESULTS}

\section{Patients' characteristics}

The CRP level was determined in 64 (39\%) among 163 patients diagnosed with NSCLC who received chemotherapy between April 2002 and January 2005, and in all 220 consecutive patients between January 2005 and 15 September 2008. In all, 13 patients were excluded because of missing covariate values and 2 because of outlier values for platelet $\left(<40000 \times 10^{-6} 1^{-1}\right)$. Therefore, 269 $(70 \%)$ out of 383 patients were available for the analyses. Table 1 presents baseline characteristics of the patients included in the study. For all 269 study subjects, we had complete data on all covariates shown in Table 1 , with the exception of five $(2 \%)$ patients, who had missing data on calcium. Excluded patients had similar characteristics, except that they received single-agent regimen of chemotherapy more frequently than the patients included in the analyses (results not shown).

The minimum follow-up was 3 days (acute complications after initiation of chemotherapy), with a median follow-up of 8.6 months and median survival of 9.2 months. During the follow-up, $211(78.4 \%)$ patients died. No patients were lost to follow-up.

\section{Univariate survival analyses and multivariable model building}

Left part of Table 2 summarises the results of separate, univariate Cox's PH models, each evaluating one of the potential predictors. The third column of Table 2 shows that in the univariate Cox's $\mathrm{PH}$ models, all variables, with the exception of age and tumour pathology, had statistically significant $(P<0.05)$ or marginally significant $(0.05<P<0.10)$ crude unadjusted associations with the hazard. However, the two last columns show that, in univariate flexible spline-based models, either the $\mathrm{PH}$ or the linearity assumptions were violated $(P<0.05$ for the respective test) for several variables.

In the multivariable Cox's PH regression analyses, the stepwise selection procedure eliminated age, sex, tumour pathology, calcium, haemoglobin, platelet, and percentage of weight lost, as their adjusted $\mathrm{PH}$-linear effects were all statistically nonsignificant $(P>0.05)$. In the multivariable Cox's PH model, the constant-overtime, linear effects of albumin and ALP were also nonsignificant (third column of Table 3 ). However, both variables were kept in the final multivariable models because of their significant effects in respective flexible spline-based models, estimated at the step 2 of the model building process (see 'Statistical analyses'). As shown in the two last columns of Table 3, while adjusted for other predictors selected into the final multivariable models, both time-dependent $(P<0.001)$ and non-linear $(P=0.024)$ effects of albumin, as well as the non-linear effect of ALP $(P=0.034)$, were all significant.

\section{Final multivariable Cox's PH model}

The left part of Table 3 summarises the results of the final multivariable Cox's PH model. C-reactive protein was found to be a very significant independent predictor of survival, with a $11 \%$ increase in the risk of death for each doubling of its value (adjusted $\mathrm{HR}=1.11,95 \% \mathrm{CI}: 1.03-1.20, P=0.008)$. Among binary prognostic factors, smoking (ever $v s$ never), higher baseline cancer stage (IIIB + pleural effusion vs IIIA/IIIB), and type of chemotherapy regimen (single- $v s$ double-agent regimen) were all independently associated with significantly higher mortality, while higher performance status (ECOG 2 vs $0-1$ ) showed a trend toward worse prognosis $(P=0.086)$. In addition to CRP, other significant continuous predictors were $\mathrm{LDH}$, with more than a two-fold, $116 \%$ increase in the risk of death for each doubling of its value, lower lymphocyte count, and higher ANC (third column of Table 3). In contrast to the univariate Cox's $\mathrm{PH}$ model (third column of 
Table 2 Results of univariate Cox's PH models $(N=269)^{\text {a }}$

\begin{tabular}{|c|c|c|c|c|}
\hline Variables & $\begin{array}{c}\text { HR } \\
(95 \% \mathrm{Cl})^{\mathrm{b}}\end{array}$ & $\begin{array}{l}P \text {-value for test of } \\
\text { no association }\end{array}$ & $\begin{array}{l}P \text {-value for } \\
\text { test of PH }\end{array}$ & $\begin{array}{c}P \text {-value for } \\
\text { test of linearity }\end{array}$ \\
\hline Age & $1.009(0.996,1.021)$ & 0.180 & 0.119 & 0.268 \\
\hline $\mathrm{ECOG}^{c}$ performance status: $(2$ vs $0-1)$ & $2.034(1.488,2.782)$ & $<0.001$ & 0.047 & N/A \\
\hline Smoking status: (ever vs never) & $1.934(1.278,2.927)$ & 0.002 & 0.253 & N/A \\
\hline Chemotherapy type: (single vs double) & $1.845(1.353,2.515)$ & $<0.001$ & 0.153 & N/A \\
\hline Squamous cell carcinoma & $0.934(0.611,1.428)$ & 0.753 & 0.610 & \\
\hline Undifferentiated carcinoma & $1.283(0.858,1.917)$ & 0.225 & 0.193 & \\
\hline Log $_{2}$ CRP: (per doubling of CRP values) & $1.175(1.102,1.252)$ & $<0.001$ & 0.002 & 0.078 \\
\hline Albumin: (per $\downarrow^{d}$ of $|g|^{-1}$ ) & $1.098(1.061,1.136)$ & $<0.001$ & $<0.001$ & 0.190 \\
\hline $\log _{2}$ LDH: (per doubling of LDH values) & $2.336(1.877,2.909)$ & $<0.001$ & 0.130 & 0.093 \\
\hline Calcium: (per $\uparrow^{\mathrm{e}}$ of $\mid \mathrm{mmol} \mathrm{I}^{-1}$ ) & $2.025(0.897,4.571)$ & 0.089 & 0.533 & 0.137 \\
\hline Alkaline phosphatase: (per $\uparrow$ of $\left.10 \cup I^{-1}\right)$ & $1.044(1.020,1.068)$ & $<0.001$ & 0.443 & 0.930 \\
\hline
\end{tabular}

Abbreviations: $\mathrm{CRP}=\mathrm{C}$-reactive protein; $\mathrm{LDH}=$ lactate dehydrogenase; $\mathrm{PH}=$ proportional hazard. $\mathrm{N} / \mathrm{A}$ : the test of linearity is not applicable to categorical covariates. ${ }^{\text {a }} \mathrm{N}=269$ for all variables except for calcium for which $N=264$. ${ }^{b}$ Unadjusted hazard ratio (HR) and $95 \%$ confidence interval $(95 \% \mathrm{Cl}) .{ }^{\mathrm{C}}$ Eastern cooperative oncology group. ${ }^{\mathrm{d}} \downarrow$ : decrease. ${ }^{\mathrm{e}} \uparrow$ : increase.

Table 3 Results of the multivariable Cox's PH model $(N=269)$

\begin{tabular}{|c|c|c|c|c|}
\hline Variables & $\begin{array}{c}\text { HR } \\
(95 \% \mathrm{Cl})^{\mathrm{a}}\end{array}$ & $\begin{array}{l}\text { P-value for test of } \\
\text { no association }\end{array}$ & $\begin{array}{l}P \text {-value for } \\
\text { test of PH }\end{array}$ & $\begin{array}{c}\text { P-value for } \\
\text { test of linearity }\end{array}$ \\
\hline Stage: (IIIB+pleural effusion/4 vs IIIA/IIIB) & $1.815(1.268,2.597)$ & 0.001 & 0.204 & $N / A$ \\
\hline Chemotherapy type: (single vs double) & $1.539(1.082,2.188)$ & 0.016 & 0.067 & $N / A$ \\
\hline $\log _{2}$ CRP: (per doubling of CRP values) & $1.108(1.027,1.196)$ & 0.008 & 0.039 & 0.130 \\
\hline Albumin: (per $\downarrow^{c}$ of $\left.|g|^{-1}\right)$ & $1.015(0.974,1.058)$ & 0.485 & $<0.001$ & 0.024 \\
\hline Neutrophil counts: (per $\uparrow$ of $\left|\times 10^{9}\right|^{-1}$ ) & $1.082(1.037,1.129)$ & $<0.001$ & 0.027 & 0.041 \\
\hline Lymphocytes: (per $\downarrow$ of $\left|\times 10^{9}\right|^{-1}$ ) & $1.307(1.050,1.626)$ & 0.016 & 0.550 & 0.460 \\
\hline Deviance e & 1902.2 & & & \\
\hline $\mathrm{AIC}$ & 1922.2 & & & \\
\hline
\end{tabular}

Abbreviations: $\mathrm{AIC}=$ Akaike's information criterion; $\mathrm{CRP}=\mathrm{C}$-reactive protein; $\mathrm{LDH}=$ lactate dehydrogenase; $\mathrm{PH}=$ proportional hazard. $\mathrm{N} / \mathrm{A}$ : the test of linearity is not applicable to categorical covariates. ${ }^{a}$ Adjusted hazard ratio (HR) and $95 \%$ confidence interval $(95 \% \mathrm{Cl})$. ${ }^{b}$ Eastern cooperative oncology group. ${ }^{c} \downarrow$ : decrease. ${ }^{\mathrm{d}} \uparrow$ : increase. eDeviance $=-2 * \log$-likelihood.

Table 2), in the final multivariable Cox's PH model, albumin was not a significant predictor of mortality $(\mathrm{HR}=1.02,95 \% \mathrm{CI}$ : $0.97-1.06, P=0.485)$.

\section{Final flexible spline-based multivariable model}

At the step 3 of the model building process, we first built a large flexible spline-based model that included all 'non-parametric' (time-dependent and/or non-linear) effects that were identified as significant $(P<0.05)$ in either of the two rightmost columns of Table 3. After having adjusted for all other non-parametric effects included in this large model, both the time-dependent and the non-linear effects of ANC, as well as the non-linear effect of ALP, became nonsignificant (data not shown). Accordingly, all three effects were eliminated from the final flexible spline-based model. In contrast, the time-dependent and non-linear effects of both albumin and $\log _{2}$ CRP retained their statistical significance and, thus, were kept in the final flexible model.
Table 4 summarises the results of the final flexible splinebased multivariable model, with the same predictors as the multivariable Cox's $\mathrm{PH}$ model shown in Table 3. In the final flexible spline-based model, only $\log _{2}$ CRP and albumin had significant non-parametric effects. For the eight other covariates, the adjustment for non-linear and time-dependent effects of $\log _{2}$ CRP and albumin do not materially alter the HRs, relative to the Cox's $\mathrm{PH}$ model, and all conclusions regarding their statistical significance are the same in both final multivariable models (Table 3 vs Table 4).

For $\log _{2}$ CRP and albumin, the last column of Table 4 shows $P$-values for the 5-df tests of the overall statistical significance of their adjusted effects on the hazard. Both $P$-values are below 0.01 , indicating that, after having accounted for their non-linear and time-dependent effects, both variables have highly significant associations with mortality. For albumin, this finding is striking in contrast to its completely nonsignificant effect in the multivariable Cox's PH model $(P=0.485$ in Table 3$)$. 
Table 4 Results of the flexible spline-based model $(N=269)$

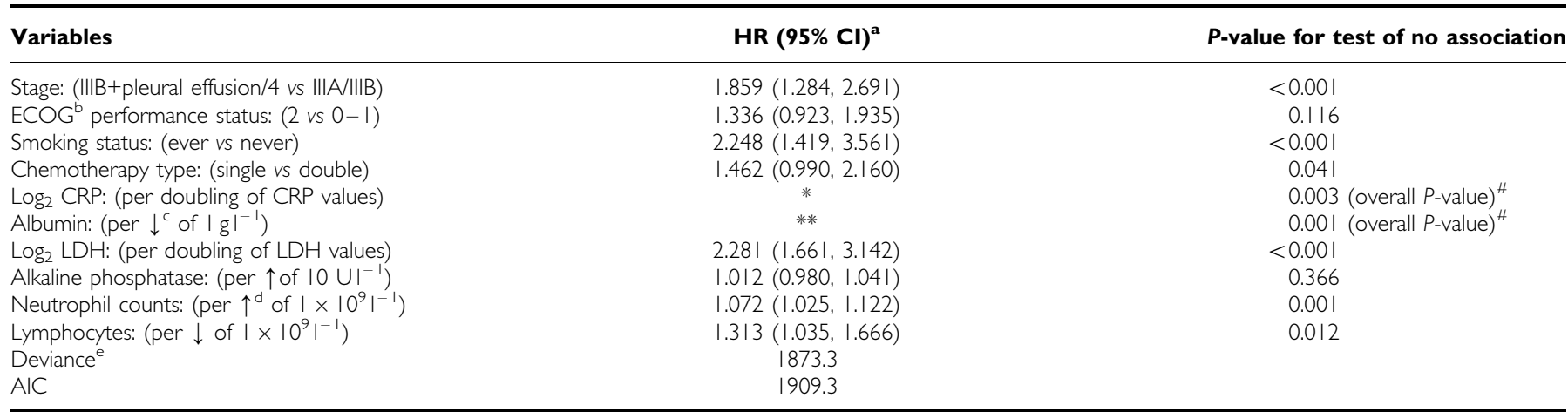

Abbreviations: AIC = Akaike's information criterion; $\mathrm{CRP}=\mathrm{C}$-reactive protein; $\mathrm{LDH}=$ lactate dehydrogenase. ${ }^{\mathrm{a}} \mathrm{Adjusted}$ hazard ratio $(\mathrm{HR})$ and $95 \%$ confidence interval $(95 \% \mathrm{Cl})$ ${ }^{b}$ Eastern cooperative oncology group. ${ }^{c} \downarrow$ : decrease. ${ }^{d} \uparrow$ : increase. ${ }^{e}$ Deviance $=-2$ * log-likelihood. *Both the time-dependent $(P=0.033)$ and non-linear $(P=0.0 \mathrm{I} 5)$ effects were significant. The estimated non-linear effects, at selected follow-up times, are shown in Figure 2 . ***B Both the time-dependent $(P=0.000 \mathrm{I})$ and non-linear $(P=0.038)$ effects were significant. The estimated non-linear effects, at selected follow-up times, are shown in Figure 3 . " $P$-value for a likelihood ratio test, with 5 degrees of freedom, of the null hypothesis of no association, obtained by comparing the deviances of (i) a flexible model where both time-dependent and non-linear effects of a given variable are modeled by splines, vs (ii) a simpler 'reduced' model, which does not include the variable being tested (see the section on "Statistical analyses" for details of the test).

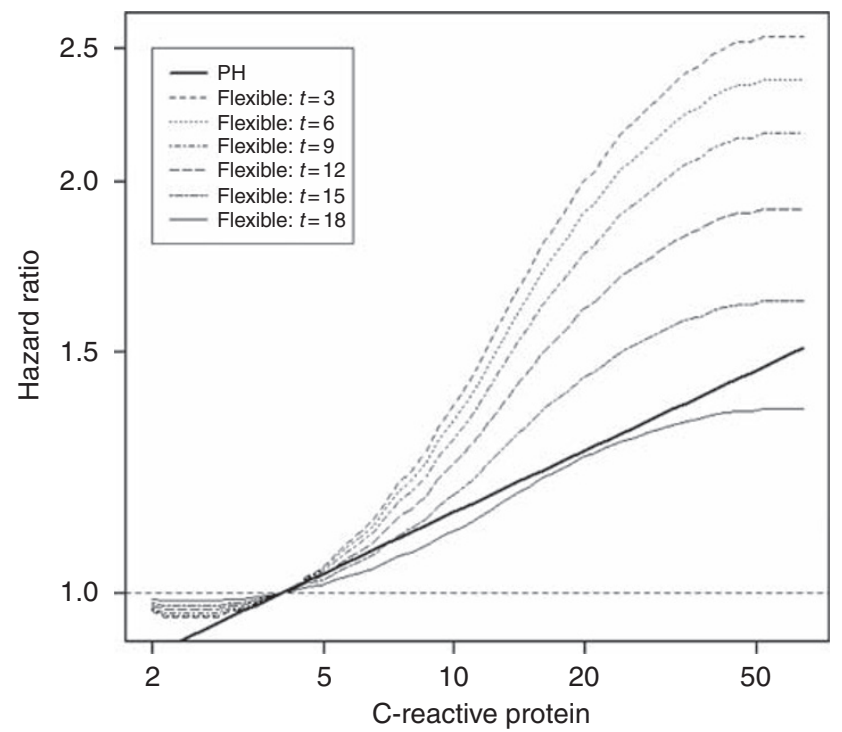

Figure 2 Results of the Cox's PH and flexible spline-based multivariable modeling of the effect of CRP on survival. The bold line represents the linear estimate from the Cox's PH model. The curves correspond to the flexible spline estimates at different times from 3 months $(t=3)$ to 18 months $(t=18)$ after the initiation of chemotherapy. Each curve shows how the adjusted hazard ratio at the corresponding time, relative to the value of $4 \mathrm{mg}^{-1}$, changes with increasing value of $\mathrm{C}$-reactive protein.

In the final flexible spline-based multivariable model, $\log _{2} \mathrm{CRP}$ had a statistically significant time-dependent effect, as the $\mathrm{PH}$ assumption was rejected $(P=0.033)$, and a significant non-linear relationship with the logarithm of the mortality hazard (linearity rejected at $P=0.015$ ). Figure 2 shows the implications of the joint violation of the $\mathrm{PH}$ and linearity assumptions for the predictive ability of baseline CRP. The thick black line in Figure 2 shows the linear, constant-over-time effect of increasing the baseline CRP, as estimated in the multivariable Cox's PH model. As the Cox's model imposes the $\mathrm{PH}$ assumption, this linear effect is assumed to apply to all times during the follow-up. In contrast, the dashed curves in Figure 2 represents the effect of baseline CRP estimated in the flexible spline-based model, for different times elapsed because the CRP was measured. Each curve shows how the current risk of allcause mortality, at that specific follow-up time, changes with the increasing value of the baseline CRP. The fact that the curves rise steeply confirms that higher CRP is associated with an important risk increase. However, the slope of the curves differs across different intervals of CRP values: the risk increases are much steeper between 5 and $30 \mathrm{mgl}^{-1}$ than outside this interval (Figure 2), which reflects the important non-linear effect of CRP. The steep increases in mortality in the middle range of CRP values indicate also that any dichotomisation of these values, regardless of the potential cut-off, will entail an important loss of information, as patients with substantially different actual risks will be predicted to have the same risk. For example, at 3 months of follow-up, CRP $=50 \mathrm{mgl}^{-1}$ is associated with an almost two-fold risk increase relative to $\mathrm{CRP}=11 \mathrm{mgl}^{-1}$ (the top curve in Figure 2), even if both values are above the conventional CRP cut-off of $10 \mathrm{mgl}^{-1}$.

The curves in Figure 2 become gradually less steep as the followup time increases. This reflects the significant time-dependent effect of CRP and indicates that the effect of high baseline CRP on mortality gradually decreases with increasing time since its initial measurement. Indeed, Figure 2 suggests that the baseline CRP retains some predictive value only for the initial $12-18$ months. In sensitivity analyses, we investigated the potential time-dependent effect of the dichotomised CRP, with the conventional $>10 \mathrm{mgl}^{-1}$ cut-off (Mahmoud and Rivera, 2002; Maltoni et al, 2005; McMillan, 2008). As in the primary analyses, with the non-linear effect of continuous CRP, the $\mathrm{PH}$ hypothesis was rejected for binary CRP $(P=0.035)$. Furthermore, the time-dependent estimate for the dichotomised CRP was very similar to that for continuous CRP, with a gradual decrease of its effect over time (data not shown). In the first 8 months after the initiation of chemotherapy, patients with baseline $\mathrm{CRP}>10 \mathrm{mgl}^{-1}$ had a two-fold higher mortality than patients below the cut-off, with the same values of all other prognostic factors, but 2 years after diagnosis their relative risk increase was as small as $20 \%$ (data not shown).

In the final flexible spline-based model, albumin also had significant non-linear $(P=0.038)$ and, especially, time-dependent effects $(P<0.001)$. The three dashed curves in Figure 3, constructed similarly to Figure 2, show how the hazard of mortality changes with increasing value of baseline albumin, respectively, at 3,6, and 9 months of follow-up. At 3 months, patients with low initial albumin, between 25 and $30 \mathrm{mgl}^{-1}$, have approximately a two-fold higher risk of death than those with the sample mean value of approximately $40 \mathrm{mgl}^{-1}$ (the steepest curve, at the top of Figure 3). In contrast, by 9 months of follow-up the curve becomes very flat, indicating that initial albumin value has no predictive 


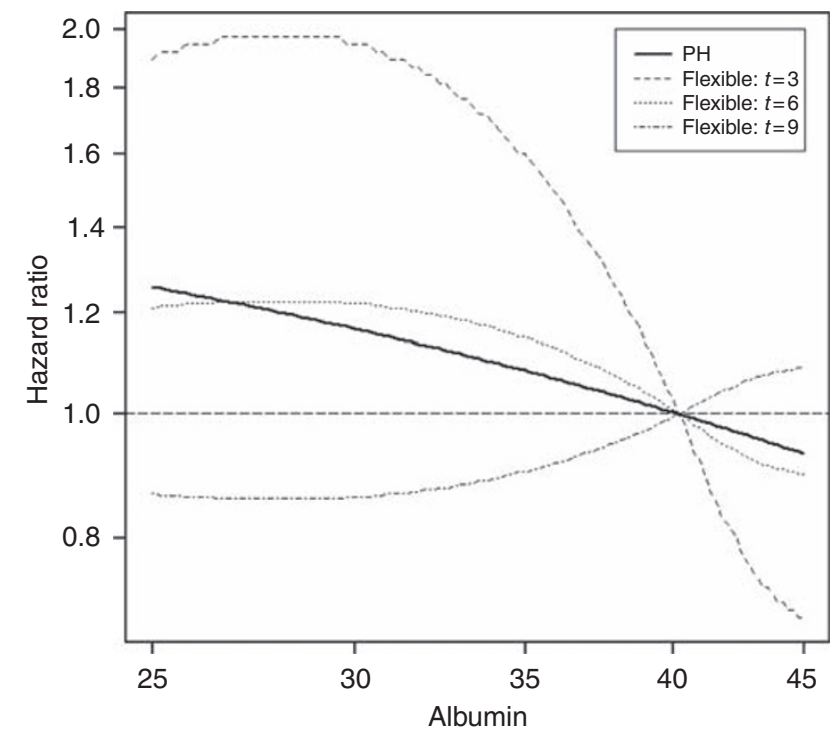

Figure 3 Results of the Cox's PH and flexible spline-based multivariable modeling of the effect of albumin on survival. The bold line represents the linear estimate from the Cox's PH model. The curves correspond to the flexible spline estimates at different times from 3 months $(t=3)$ to 9 months $(t=9)$ after the initiation of chemotherapy. Each curve shows how the adjusted hazard ratio at the corresponding time, relative to the value of $40 \mathrm{mgl}^{-1}$, changes with decreasing value of albumin.

value at or beyond 9 months after it was measured (the curve at the bottom of Figure 3). This sharp decrease in the prognostic value of baseline albumin during the follow-up reflects its very significant time-dependent effect $(P<0.001)$. This also explains why albumin was completely nonsignificant in the multivariable Cox's PH model $(P=0.485)$, in which its estimated effect was a priori constrained to be constant over time. By imposing this constraint, totally inconsistent with the actual, very significant time-dependent effect of albumin, the Cox's $\mathrm{PH}$ estimate, represented by a thick black line in Figure 3, suggested decreasing albumin had only a very weak effect on increased mortality hazard. This masked the important short-term increase in risk for patients with low baseline albumin, below $35 \mathrm{mgl}^{-1}$, shown by the flexible spline estimate, for $t=3$ months, in Figure 3.

\section{Goodness-of-fit and model validation}

The final flexible spline-based multivariable model, which accounted for the significant time-dependent and non-linear effects of both $\log _{2}$ CRP and albumin, yielded an important improvement in the fit to our data, as reflected by the much lower value of AIC than for the multivariable Cox's $\mathrm{PH}$ model, with the same variables (AIC $=1909.3$ in Table 4 vs 1922.2 in Table 3).

The five-fold cross-validation confirmed that the flexible splinebased multivariable model could be expected to better predict the relative risks in an independent sample from a similar population, as its cross-validated deviance was substantially lower than for the Cox's PH model with the same predictor variables $(1230.5$ vs 1263.6).

\section{DISCUSSION}

We have re-assessed the role of the CRP and other biomarkers in the prognosis of NSCLC. We considered a larger number of potential prognostic factors (Table 1) than most previous studies of NSCLC mortality (Brundage et al, 2002; Watine et al, 2006) and tested the important assumptions underlying the Cox's PH model, on which those studies relied (Kato et al, 2000; Forrest et al, 2003,
2004). To this end, we have used a new, flexible spline-based model that permitted testing of the conventional $\mathrm{PH}$ and linearity assumptions, and accounting for their violations (Abrahamowicz and MacKenzie, 2007). The results confirmed the advantages of such flexible modeling by revealing statistically and clinically significant violations of both assumptions for CRP and albumin. Below, we explain in detail the important clinical implications of accounting for these violations for the prognosis of individual patients survival in NSCLC.

The multivariable Cox's $\mathrm{PH}$ model in Table 3 suggested that the risk of mortality increased by approximately $11 \%$ for each doubling of CRP, and the underlying linearity assumption would imply that the same increase applied to the comparisons of CRP of (a) $4 v s 8 \mathrm{mgl}^{-1}$, as (b) $8 v s 16 \mathrm{mgl}^{-1}$. Yet, our flexible spline-based model indicated that the linearity assumption was violated $(P=0.015)$, and that the actual risk increase was much steeper between CRP values of 8 and $16 \mathrm{mgl}^{-1}$ (Figure 2). A majority of the published cancer prognostic studies dichotomise CRP at $10 \mathrm{mgl}^{-1}$ (Mahmoud and Rivera, 2002; Maltoni et al, 2005; McMillan, 2008), and the Glasgow Prognostic Score uses the same cut-off (Forrest et al, 2003). Yet, Figure 2 shows that while patients with $\mathrm{CRP}>10 \mathrm{mgl}^{-1}$ are, on average, at much higher risk than those below this cut-off, the risk of death increases continuously between 4 and $50 \mathrm{mgl}^{-1}$, with steepest increases between 8 and $20 \mathrm{mgl}^{-1}$. Consequently, at 3 months of follow-up, CRP $=50 \mathrm{mgl}^{-1}$ is associated with almost two-fold higher risk than $\mathrm{CRP}=11 \mathrm{mgl}^{-1}$, even if both values fall above the conventional $10 \mathrm{mgl}^{-1}$ cut-off. Thus, our flexible, non-linear estimate of the CRP effect helps avoiding inaccurate risk assessment and loss of prognostic information, which would be induced by conventional dichotomised or linear estimates (Ramsay, 1988; Hastie and Tibshirani, 1990; Royston and Altman, 1994; Greenland, 1995; Abrahamowicz et al, 1997; Benedetti and Abrahamowicz, 2004).

Second, the PH assumption imposes that in the Cox's PH model the relative risk associated with each prognostic factor remains constant during the entire follow-up period. Thus, the HR between any two baseline CRP values is a priori constrained to be the same at the time of its measurement as, for example, 12 months later. However, our flexible spline-based analyses rejected the $\mathrm{PH}$ hypothesis for CRP, and indicated that it retains the prognostic value for approximately a year (Figure 2). The gradual loss of prognostic ability of the baseline CRP may occur because (a) some patients, with high initial CRP value, for unknown reasons, responded to chemotherapy better than others, (b) in patients with oesophageal squamous cell cancer, CRP polymorphism may gradually modify tumour progression (Motoyama et al, 2009), and/or (c) correlation between the baseline CRP and its current values decreases over time. Future studies should examine the latter conjecture, by using time-dependent covariates to model the effect of updated CRP values.

For albumin, our flexible spline-based model revealed a dramatic decrease in its prognostic value over time $(P<0.001)$. Figure 3 shows that low baseline albumin, below $30 \mathrm{mgl}^{-1}$, is associated with a statistically significant and clinically important mortality risk increase in the first 3 months of follow-up. However, the effect of baseline albumin on mortality rapidly declines thereafter, and becomes practically null after approximately 9 months. This rapid loss of prognostic value explains why albumin was completely nonsignificant in the multivariable Cox's PH model $(P=0.485)$, which estimates the average relative risks across the follow-up (Abrahamowicz et al, 1996).

In conclusion, the Cox's model, by imposing the incorrect $\mathrm{PH}$ assumption, failed to identify albumin as an important early prognostic factor for NSCLC mortality. Clinical observations suggested that low albumin might indicate an unfavourable prognosis, especially in the near future (Nixon et al, 1980; Hill, 1987; Heys et al, 1992). Still, our flexible spline-based model showed the statistical significance of time-dependent changes in the effect of 
albumin, and detected the dramatic effect of low baseline albumin on NSCL mortality in the next 3-4 months. Thus, flexible analyses enhanced both the validity and the accuracy of conclusions regarding prognostic value of albumin.

The implications of the violation of the conventional $\mathrm{PH}$ and linearity assumptions are relevant for clinicians. By accounting for the time-dependent changes in the effects of both markers, the flexible model helps them to realise that the risk of death associated with high CRP and low albumin is very high for the first 6-12 months after diagnosis. Furthermore, by accounting for important non-linearities of the relationships between the marker values and the hazard, the flexible model improves substantially clinicians' ability to identify high-risk subgroups. For example, the Cox's PH model incorrectly suggests that only a small increase $(23 \%)$ of risk of death occurs with an increase of baseline CRP from 4 to $16 \mathrm{mgl}^{-1}$, while in the more accurate, flexible model it is associated with a much higher $(80 \%)$ risk increase. Such objective prognostic information, when provided in the timely manner, may influence patients' crucial decisions, possibly making them more likely to decline life-prolonging therapy and to opt for comfort care (Weeks et al, 1998b), which is too often not discussed in the first 4-7 months after diagnosis (Huskamp et al, 2009). It is noteworthy that disclosure of accurate prognostic information may not be associated with the loss of hope, even in a dramatic situation (Mack et al, 2007).

Our analyses confirm the higher baseline cancer stage as a powerful independent prognostic factor for NSCLC mortality (Martin et al, 1999; Forrest et al, 2003). In contrast, the adjusted effect of performance status did not reach statistical significance, possibly because of limited statistical power and/or misclassification, as physicians tend to underestimate the performance status (Ando et al, 2001). As in other studies, smoking (Carney, 2002) and neutrophil count (Watine, 2000) were associated with increased mortality. In our study, LDH was a powerful prognostic factor: the hazard increased more than two-fold with each doubling of LDH. The effect of LDH was independent of CRP and albumin, suggesting that LDH affects survival through a biological process other than inflammation. Evidence shows that upregulation of LDH-5 is common in NSCLC and, when associated with overexpression of the Hypoxia-inducible factor 1, induces a strong anaerobic glycolytic metabolism and a reduced dependence on oxygen, resulting in decreased survival (Koukourakis et al, 2003). Of interest, the $\mathrm{PH}$ assumption was not violated for $\mathrm{LDH}$, suggesting that this pathway remains unchanged over time.

Our findings regarding both CRP and albumin confirm the paramount importance of testing the assumptions underlying the very popular Cox's PH model (Altman et al, 1995). The striking differences between the estimated effects of both variables obtained with the Cox's PH model $v s$ the flexible spline-based model are reflected in Figures 2 and 3. Overall, our results illustrate potentially serious clinical and research implications of imposing these assumptions a priori, which may lead to a failure to identify important prognostic factors, such as albumin in our study, inaccurate identification of high-risk groups, or spurious contradictions between the results of short- $v s$ long-term prognostic studies. These results are in line with several other clinical and methodological studies indicating important violations of $\mathrm{PH}$ and/or linearity hypotheses (Ramsay, 1988; Sleeper and Harrington, 1990; Hastie and Tibshirani, 1990; Royston and Altman, 1994; Greenland, 1995; Rothman et al, 1995; Abrahamowicz et al, 1997; Benedetti and Abrahamowicz, 2004; Royston et al, 2006; Spix et al, 2008). The PH assumption can be tested with simple parametric or non-parametric tests available in a standard statistical software packages (Wei, 1984). If the PH hypothesis is rejected, the time-dependent effect of the prognostic factor can be estimated with flexible survival models, using either fractional polynomials (Sauerbrei et al, 2007) or splines (Gray, 1992; Hess, 1994; Kooperberg et al, 1995; Abrahamowicz et al,
1996; Abrahamowicz and MacKenzie, 2007), including the method incorporated in the R package (Grambsh and Therneau, 1994). To test the linearity hypothesis and estimate non-linear effects of continuous predictors on the hazard, one can use splines (Gray, 1992; Kooperberg et al, 1995; Abrahamowicz and MacKenzie, 2007; Remontet et al, 2007), or fractional polynomials (Royston and Altman, 1994; Sauerbrei et al, 2007; Royston and Sauerbrei, 2008), incorporated in STATA (StataCorp LP, College Station, TX, USA), $\mathrm{R}$ ( $\mathrm{R}$ Foundation for Statistical Computing, Vienna, Austria) package mfp (Sauerbrei et al, 2006), and a SAS (SAS Institute Inc.) macro (Sauerbrei et al, 2006).

The flexibility of modeling offered by splines and fractional polynomials ensures that the estimated effects are represented by smooth functions rather than by 'step-functions', resulting from categorisation of continuous variables or of the follow-up time, which impose clinically implausible 'jumps' in the risk, at arbitrary selected covariate or time values. Furthermore, the flexibility of these modeling tools permits an accurate recovery of a large variety of curves with a single estimator, thus, avoiding the loss of efficiency and inaccurate statistical inference induced when the analyst uses several alternative parametric transformations of the covariate or time axis (Hastie and Tibshirani, 1990; Quantin et al, 1999; Mahmud et al, 2006).

Our study has some limitations. First, we relied on retrospective analyses of data collected prospectively on a small number of patients, for a clinical quality assessment program, in a single centre. Prospective collection increases data accuracy and reduces risk of selection or misclassification biases. However, as in other prospective studies, self-reported data on weight loss may be affected by recall bias. This might have attenuated the estimated effect of weight loss and explain its nonsignificance in the multivariable models. Second, the study population does not include all the consecutive patients observed in our POC clinic between January 2002 and January 2005 when only some physicians did test their patients for CRP. However, it is unlikely that patients of different physicians had different characteristics, because POC physicians act as a group practise. Indeed, there were no clinically relevant differences between included and excluded patients on any measured variable, except for the frequency of single $v s$ double chemotherapy regimen. During the study period, double regimen was considered advantageous for the higher risk patients (Lilenbaum et al, 2000; Lilenbaum, 2003).

Finally, because our data are limited to a single clinical centre, the generalisability of our results and conclusions needs to be assessed in an independent study. This will also permit a direct 'external' validation of our flexible spline-based model. Still, the 'internal' cross-validation, which approximates validation in an independent sample (Harrell, 2001; Binquet et al, 2008), indicated that our flexible spline-based model substantially improved prediction over the Cox's PH model.

Among numerous biological markers being currently investigated (Brundage et al, 2002), recent reports suggest that neuronspecific enolase (NSE) may be an independent prognostic factor for survival in NSCLC (Maeda et al, 2000; Jacot et al, 2001; Ferrigno et al, 2003). However, NSE could not be included in our analyses, as in our institution it is used infrequently and only for diagnostic purposes (Hatzakis et al, 2002). Future research should assess if adjusting for NSE may affect the results of flexible analyses of NSCLC mortality.

\section{CONCLUSION}

Our study has important clinical and research implications. From a research perspective, it illustrates the importance of using flexible survival models to both test the assumptions underlying the popular Cox's PH model and accurately estimate the relative risks that may change considerably during the follow-up. 
From a clinical perspective, it shows that while both albumin and CRP are important prognostic factors for NSCLC mortality, in this small retrospective study, their prognostic value does not extend beyond, respectively, 6 or 12 months after the initial measurement.

\section{ACKNOWLEDGEMENTS}

MA is a James McGill Professor at McGill University. This research was supported by grants from the Terry Fox Research Institute and the Canadian Institutes of Health Research (CIHR) grant MOP-8127.

\section{REFERENCES}

Abrahamowicz M, Ciampi A (1991) Information theoretic criteria in nonparametric density estimation: bias and variance in the infinite dimensional case. Comput Stat Data Anal 12: 239-247

Abrahamowicz M, du Berger R, Grover SA (1997) Flexible modeling of the effects of serum cholesterol on coronary heart disease mortality. Am J Epidemiol 145(8): 714-729

Abrahamowicz M, MacKenzie T, Esdaile JM (1996) Time-dependent hazard ratio: modeling and hypothesis testing with application in lupus nephritis. J Am Stat Assoc 91(436): 1432-1439

Abrahamowicz M, MacKenzie TA (2007) Joint estimation of timedependent and non-linear effects of continuous covariates on survival. Stat Med 26(2): $392-408$

Akaike H (1974) New Look at Statistical-Model Identification. IEEE Trans Automatic Control AC 19(6): 716-723

Altman DG, De Stavola BL, Love SB, Stepniewska KA (1995) Review of survival analyses published in cancer journals. $\mathrm{Br} J$ Cancer 72(2): $511-518$

Ando M, Ando Y, Hasegawa Y, Shimokata K, Minami H, Wakai K, Ohno Y, Sakai S (2001) Prognostic value of performance status assessed by patients themselves, nurses, and oncologists in advanced non-small cell lung cancer. Br J Cancer 85(11): $1634-1639$

Benedetti A, Abrahamowicz M (2004) Using generalized additive models to reduce residual confounding. Stat Med 23(24): 3781-3801

Berry SR (2008) Just say die. J Clin Oncol 26(1): 157-159

Binquet C, Abrahamowicz M, Astruc K, Faivre J, Bonithon-Kopp C, Quantin C (2009) Flexible statistical models provided new insights into the role of quantitative prognostic factors for mortality in gastric cancer. $J$ Clin Epidemiol 62(3): 232-240

Binquet C, Abrahamowicz M, Mahboubi A, Jooste V, Faivre J, BonithonKopp C, Quantin C (2008) Empirical study of the dependence of the results of multivariable flexible survival analyses on model selection strategy. Stat Med 27(30): 6470-6488

Brundage MD, Davies D, Mackillop WJ (2002) Prognostic factors in non-small cell lung cancer - A decade of progress. Chest 122(3): $1037-1057$

Carney DN (2002) Lung cancer - time to move on from chemotherapy. $N$ Engl J Med 346(2): 126-128

Cox DR (1972) Regression models and life-tables. J R Stat Soc Series B-Stat Methodology 34(2): 187-220

Ferrigno D, Buccheri G, Giordano C (2003) Neuron-specific enolase is an effective tumour marker in non-small cell lung cancer (NSCLC). Lung Cancer 41(3): 311-320

Forrest LM, McMillan DC, McArdle CS, Angerson WJ, Dunlop DJ (2003) Evaluation of cumulative prognostic scores based on the systemic inflammatory response in patients with inoperable non-small-cell lung cancer. Br J Cancer 89(6): $1028-1030$

Forrest LM, McMillan DC, McArdle CS, Angerson WJ, Dunlop DJ (2004) Comparison of an inflammation-based prognostic score (GPS) with performance status (ECOG) in patients receiving platinum-based chemotherapy for inoperable non-small-cell lung cancer. $\mathrm{Br} J$ Cancer 90(9): $1704-1706$

Glare P (2005) Clinical predictors of survival in advanced cancer. J Support Oncol 3(5): $331-339$

Grambsh PM, Therneau TM (1994) Proportional hazrads tests and diagnostics based on weighted residuals. Biometrika 81: $515-526$

Gray RJ (1992) Flexible methods for analyzing survival data using splines, with applications to breast cancer prognosis. J Am Stat Assoc 87: $942-951$

Greenland S (1995) Dose-response and trend analysis in epidemiology: alternatives to categorical analysis. Epidemiology 6(4): 356-365

Harrell F (2001) Regression Modeling Strategies, with Applications to Linear Models, Logistic Regression, and Survival Analysis. Springer: New York, NY, USA
Hastie TJ, Tibshirani RJ (1990) Generalized Additive Models. Chapman \& Hall/CRC: New York, NY, USA

Hatzakis KD, Froudarakis ME, Bouros D, Tzanakis N, Karkavitsas N, Siafakas NM (2002) Prognostic value of serum tumor markers in patients with lung cancer. Respiration 69(1): 25-29

Hess KR (1994) Assessing time-by-covariate interactions in proportional hazards regression models using cubic spline functions. Stat Med 13(10): $1045-1062$

Heys SD, Park KG, Garlick PJ, Eremin O (1992) Nutrition and malignant disease: implications for surgical practice. Br J Surg 79(7): $614-623$

Hill GL (1987) Malnutrition and surgical risk: guidelines for nutritional therapy. Ann R Coll Surg Engl 69(6): $263-265$

Huskamp HA, Keating NL, Malin JL, Zaslavsky AM, Weeks JC, Earle CC, Teno JM, Virnig BA, Kahn KL, He Y, Ayanian JZ (2009) Discussions with physicians about hospice among patients with metastatic lung cancer 7 . Arch Intern Med 169(10): 954-962

Jacot W, Quantin X, Boher JM, Andre F, Moreau L, Gainet M, Depierre A, Quoix E, Chevalier TL, Pujol JL (2001) Brain metastases at the time of presentation of non-small cell lung cancer: a multi-centric AERIO analysis of prognostic factors. Br J Cancer 84(7): 903-909

Kato K, Hitsuda Y, Kawasaki Y, Igishi T, Yasuda K, Mikami M, Watanabe M, Miyata M, Sasaki T, Shimizu E (2000) [The value of serum C-reactive protein as a survival determinant in patients with advanced non-small-cell lung cancer] (Japanese). Nihon Kokyuki Gakkai Zasshi 38(8): 575-580

Kellett J (2008) Prognostication-the lost skill of medicine. Eur J Intern Med 19(3): 155-164

Koch A, Fohlin H, Sorenson S (2009) Prognostic significance of C-reactive protein and smoking in patients with advanced non-small cell lung cancer treated with first-line palliative chemotherapy. $J$ Thorac Oncol 4(3): $326-332$

Kooperberg C, Stone CJ, Truong YK (1995) Hazard regression. J Am Stat Assoc 90: 78-94

Koukourakis MI, Giatromanolaki A, Sivridis E, Bougioukas G, Didilis V, Gatter KC, Harris AL (2003) Lactate dehydrogenase-5 (LDH-5) overexpression in non-small-cell lung cancer tissues is linked to tumour hypoxia, angiogenic factor production and poor prognosis. Br J Cancer 89(5): $877-885$

Lambert PC, Smith LK, Jones DR, Botha JL (2005) Additive and multiplicative covariate regression models for relative survival incorporating fractional polynomials for time-dependent effects. Stat Med 24(24): $3871-3885$

Lilenbaum R (2003) Management of advanced non-small-cell lung cancer in elderly populations. Clin Lung Cancer 5(3): 169-173

Lilenbaum R, Cano R, Schwartz M, Siegel L, Lutzky J, Lewis M, Krill E, Barreras L, Davila E (2000) Gemcitabine and vinorelbine in advanced nonsmall cell lung carcinoma: a phase II study. Cancer 88(3): 557-562

Mack JW, Wolfe J, Cook EF, Grier HE, Cleary PD, Weeks JC (2007) Hope and prognostic disclosure 1. J Clin Oncol 25(35): 5636-5642

Maeda T, Ueoka H, Tabata M, Kiura K, Shibayama T, Gemba K, Takigawa N, Hiraki A, Katayama H, Harada M (2000) Prognostic factors in advanced non-small cell lung cancer: elevated serum levels of neuron specific enolase indicate poor prognosis. Jpn J Clin Oncol 30(12): 534-541

Mahmoud FA, Rivera NI (2002) The role of C-reactive protein as a prognostic indicator in advanced cancer. Curr Oncol Rep 4(3): 250-255

Mahmud M, Abrahamowicz M, Leffondre K, Chaubey Y (2006) Selecting the optimal transformation of a continuous covariate in Cox's regression: Implications for hypothesis testing. Commun Stat 35(1): 27-45

Maltoni M, Caraceni A, Brunelli C, Broeckaert B, Christakis N, Eychmueller S, Glare P, Nabal M, Vigano A, Larkin P, De CF, Hanks G, Kaasa S (2005) Prognostic factors in advanced cancer patients: evidence-based clinical recommendations-a study by the Steering Committee of the European Association for Palliative Care. J Clin Oncol 23(25): $6240-6248$ 
Martin F, Santolaria F, Batista N, Milena A, Gonzalez-Reimers E, Brito MJ, Oramas J (1999) Cytokine levels (IL-6 and IFN-gamma), acute phase response and nutritional status as prognostic factors in lung cancer. Cytokine 11(1): $80-86$

McMillan DC (2008) An inflammation-based prognostic score and its role in the nutrition-based management of patients with cancer. Proc Nutr Soc 67(3): $257-262$

Motoyama S, Miura M, Hinai Y, Maruyama K, Usami S, Saito H, Minamiya Y, Satoh S, Murata K, Suzuki T, Ogawa J (2009) CRP genetic polymorphism is associated with lymph node metastasis in thoracic esophageal squamous cell cancer. Ann Surg Oncol 16(9): $2479-2485$

Nixon DW, Heymsfield SB, Cohen AE, Kutner MH, Ansley J, Lawson DH, Rudman D (1980) Protein-calorie undernutrition in hospitalized cancer patients. Am J Med 68(5): 683-690

Oken MM, Creech RH, Tormey DC, Horton J, Davis TE, McFadden ET, Carbone PP (1982) Toxicity and response criteria of the Eastern Cooperative Oncology Group. Am J Clin Oncol 5(6): 649-655

Pfister DG, Johnson DH, Azzoli CG, Sause W, Smith TJ, Baker Jr S, Olak J, Stover D, Strawn JR, Turrisi AT, Somerfield MR (2004) American Society of Clinical Oncology treatment of unresectable non-small-cell lung cancer guideline: update 2003. J Clin Oncol 22(2): 330-353

Quantin C, Abrahamowicz M, Moreau T, Bartlett G, MacKenzie T, Tazi MA, Lalonde L, Faivre J (1999) Variation over time of the effects of prognostic factors in a population-based study of colon cancer: comparison of statistical models. Am J Epidemiol 150(11): 1188-1200

Rachet B, Abrahamowicz M, Sasco AJ, Siemiatycki J (2003) Estimating the distribution of lag in the effect of short-term exposures and interventions: adaptation of a non-parametric regression spline model Stat Med 22(14): 2335-2363

Rachet B, Sasco AJ, Abrahamowicz M, Benyamine D (1998) Prognostic factors for mortality in nasopharyngeal cancer: accounting for timedependence of relative risks. Int J Epidemiol 27(5): $772-780$

Ramsay JO (1988) Monotone regression splines in action. Stat Sci 3(4): $425-441$

Remontet L, Bossard N, Belot A, Esteve J (2007) An overall strategy based on regression models to estimate relative survival and model the effects of prognostic factors in cancer survival studies. Stat Med 26(10): $2214-2228$

Rothman KJ, Moore LL, Singer MR, Nguyen US, Mannino S, Milunsky A (1995) Teratogenicity of high vitamin A intake. N Engl J Med 333(21): $1369-1373$
Royston P, Altman DG (1994) Regression using fractional polynomials or continuous covariates: parsimonious parametric modelling. Appl Stat 43: $429-467$

Royston P, Reitz M, Atzpodien J (2006) An approach to estimating prognosis using fractional polynomials in metastatic renal carcinoma. Br J Cancer 94(12): $1785-1788$

Royston P, Sauerbrei W (2008) Multivariable Model-Building: A Pragmatic Approach to Regression Analysis Based on Fractional Polynomials for Modelling Continuous Variables. John Wiley \& Sons Ltd: Chichester, West Sussex, England

Sauerbrei W, Meier-Hirmer C, Benner A, Royston P (2006) Multivariate regression model building by using fractional polynomials: description of SAS, STATA and R programs. Computing Statistics and Data Analysis 50: $3464-3485$

Sauerbrei W, Royston P, Look M (2007) A new proposal for multivariable modelling of time-varying effects in survival data based on fractional polynomial time-transformation. Biom J 49(3): $453-473$

Schrump DS, Giaccone G, Kelsey CR, Marks LB (2008) Cancer of the lung - section 2 non-small-cell lung cancer. In Cancer - Principles and Practice of Oncology, DeVita Jr VT, Lawrence ST, Rosenberg SA (eds). pp 916-917. Lippincott, Williams \& Wilkins: Philadelphia, PA

Sleeper LA, Harrington DP (1990) Regression splines in the Cox model with application to covariate effects in liver disease. J Am Stat Assoc 85: 941 - 949

Spix C, Eletr D, Blettner M, Kaatsch P (2008) Temporal trends in the incidence rate of childhood cancer in Germany 1987-2004. Int J Cancer 122(8): 1859

Watine J (2000) Prognostic evaluation of primary non-small cell lung carcinoma patients using biological fluid variables. A systematic review. Scand J Clin Lab Invest 60(4): 259-273

Watine J, Friedberg B, Nagy E, Onody R, Oosterhuis W, Bunting PS, Charet JC, Horvath AR (2006) Conflict between guideline methodologic quality and recommendation validity: A potential problem for practitioners. Clin Chem 52(1): 65-72

Weeks JC, Cook EF, O'Day SJ, Peterson LM, Wenger N, Reding D, Harrell FE, Kussin P, Dawson NV, Connors Jr AF, Lynn J, Phillips RS (1998a) Relationship between cancer patients' predictions of prognosis and their treatment preferences. JAMA 279(21): 1709-1714

Weeks JC, Cook EF, O’Day SJ, Peterson LM, Wenger N, Reding D, Harrell FE, Kussin P, Dawson NV, Connors Jr AF, Lynn J, Phillips RS (1998b) Relationship between cancer patients' predictions of prognosis and their treatment preferences 6. JAMA 279(21): 1709-1714

Wei J (1984) Testing goodness of fit for proportional hazards model with censored observations. J Am Stat Assoc 79: 649-652 